Provided for non-commercial research and education use. Not for reproduction, distribution or commercial use.

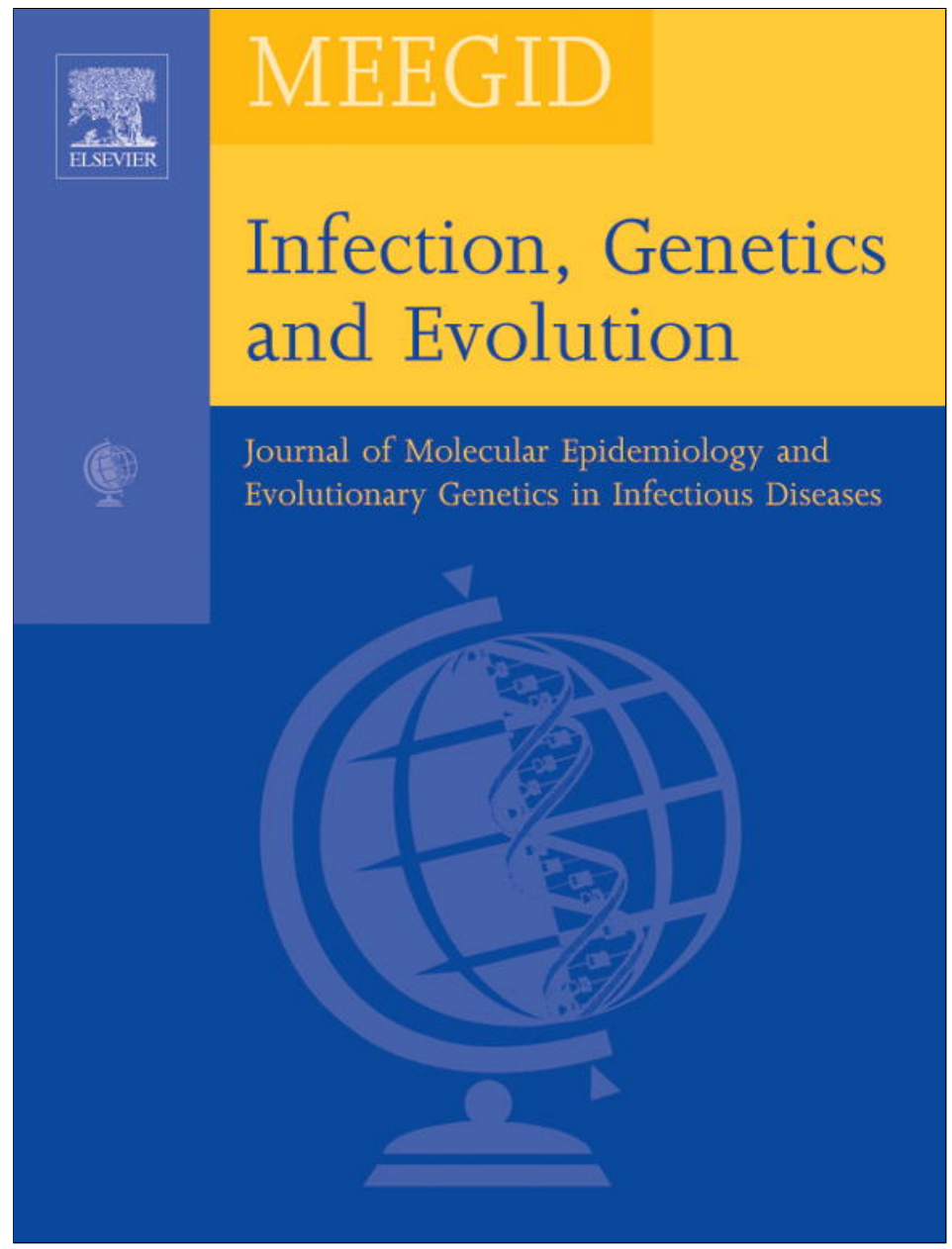

This article appeared in a journal published by Elsevier. The attached copy is furnished to the author for internal non-commercial research and education use, including for instruction at the authors institution and sharing with colleagues.

Other uses, including reproduction and distribution, or selling or licensing copies, or posting to personal, institutional or third party websites are prohibited.

In most cases authors are permitted to post their version of the article (e.g. in Word or Tex form) to their personal website or institutional repository. Authors requiring further information regarding Elsevier's archiving and manuscript policies are encouraged to visit:

http://www.elsevier.com/authorsrights 


\title{
Dynamics and molecular evolution of HIV-1 strains in Sicily among antiretroviral naïve patients
}

\author{
F. Tramuto*, C.M. Maida, F. Bonura, A.M. Perna, F. Vitale \\ Department of Sciences for Health Promotion "G. D'Alessandro" - Hygiene Section, University of Palermo, Palermo, Italy
}

\section{A R T I C L E I N F O}

\section{Article history:}

Received 14 November 2012

Received in revised form 11 February 2013

Accepted 14 February 2013

Available online 5 March 2013

\section{Keywords:}

HIV-1 subtypes

Non-B subtypes

CRF

Molecular evolution

HAART-naïve patients

\begin{abstract}
A B S T R A C T
HIV-1 subtype B is the most frequent strain in Sicily. To date, there is no available data about the genetic diversity of HIV-1 viral strains circulating in Sicily among antiretroviral (ARV) naïve subjects and the role of immigration as potential determinant of evolutionary dynamics of HIV-1 molecular epidemiology.

For this purpose, HIV-1 polymerase ( $p o l)$ sequences obtained from 155 ARV naïve individuals from 2004 to 2009 were phylogenetically analysed.

The overall rate of HIV-1 non-B infections was 31.0\% ( $n=48 / 155)$, increasing from $7.8 \%$ in $2004-2006$ to $40.9 \%$ in 2009 , and about one-third were identified as unique recombinant forms.

CRF02_AG was the prevalent non-B clade $(n=28 / 48,58.3 \%)$, while subtype C-related strains were responsible for about 30\% HIV-1 infections.

Non-B viruses strictly associated with heterosexual transmission (85.4\%) and were mostly found among immigrants (77.1\%). Phylogenetic analysis of non-B sequences found in foreign-born subjects was geographically correlated to the respective country of origin. Moreover, the detection of non-B viral variants in the autochthonous population may support an increasing genetic diversity in Sicily as well as a local circulation of HIV strains also uncommon in our country.

In Sicily, HIV-1 epidemic is still mostly attributable to the B subtype. Nevertheless, migration and population movements are progressively introducing novel HIV-1 subtypes causing a continuous increase of HIV-1 molecular dynamic at local level. Molecular surveillance is needed to monitor the genetic evolution of HIV-1 epidemic.
\end{abstract}

() 2013 Elsevier B.V. All rights reserved.

\section{Introduction}

The global HIV/AIDS epidemic is largely dominated by viruses belonging to the group $M$ of HIV-1. At present, the group $M$ is subdivided into subtypes (A-D, F-H, J and K), sub-subtypes (A1-A4, F1 and F2), and several circulating recombinant forms (CRFs) [http:// www.hiv.lanl.gov/content/sequence/HIV/CRFs/CRFs.html]. More recently, second-generation recombinants (SGRs) combining one or more CRFs with different subtypes, as well as unique recombinant forms (URFs) have been described with high prevalences in populations and in geographic areas where multiple subtype co-circulate (Geretti, 2006; Peeters et al., 2003), and referred as "geographic recombinant hotspots" (Thomson and Nájera, 2005).

\footnotetext{
* Corresponding author. Address: Department of Sciences for Health Promotion "G. D’Alessandro" - Hygiene Section, University of Palermo, 133, via del Vespro, I90127 Palermo, Italy. Tel.: +39 091 6553632; fax: +39 0916553647.

E-mail address: fabio.tramuto@unipa.it (F. Tramuto).
}

During the last decade, although some molecular forms are still "geographically confined" to the countries of their first detection (Ng et al., 2012; Liu et al., 2012; Passaes et al., 2009a,b; Ruchansky et al., 2009), non-indigenous viral variants are rapidly spreading into regions of the world historically restricted to specific HIV-1 subtypes.

In this regard, recent molecular epidemiology studies conducted in Western Europe including Italy, either in ARV-treated or -untreated patients, reported the circulation of several non-B subtypes and CRFs together with a clear increasing trend over time (Buonaguro et al., 2002; Lai et al., 2010; Monno et al., 2012, 2005; Tramuto et al., 2007, 2004).

However, the impact of HIV-1 non-B subtypes in antiretroviral naïve groups of subjects has not been previously investigated in Sicily, a Mediterranean region becoming increasingly involved in immigration influxes.

The aim of the present study was to describe the heterogeneity of HIV-1 group $M$ viruses and to investigate the evolution of subtype non-B strains by using a phylogenetic approach on HIV-1 pol sequences, among a group of antiretroviral (ARV) therapy naïve patients living in Sicily. 


\section{Materials and methods}

\subsection{Study subjects}

From February 2004 to December 2009, plasma samples from a total of 155 HIV-1 infected patients with detectable HIV plasma levels (113 native Sicilian and 42 immigrants) naïve for highly active antiretroviral therapy (HAART) $(72.3 \%$ males; median age 34.0 years; range 1-66) were consecutively collected at the AIDS Regional Reference Laboratory - University of Palermo and tested for the genotypic resistance to antiretroviral drugs as part of the pre-therapy routine procedure.

During the first visit to hospital, an individual's formal written permission to use any part of person's health data (e.g., demographic, clinical and laboratory information) in HIV epidemiology research studies was obtained from each patient or parents of children participants involved in the study. The present work was reviewed and approved by the institutional review board of the University Hospital “A.O.U.P. - P. Giaccone” of Palermo (Sicily), health data were stored according to the Italian laws on privacy, and the research was conducted following the Helsinki declaration statements.

\subsection{Pol gene PCR amplification and sequencing}

Plasma HIV-1 RNA levels were measured using the Roche Amplicor system (HIV Monitor, Roche Diagnostics Corp., Durham, NC) following the manufacturer's instructions. All plasma samples had HIV-1 RNA levels above the minimum detection limit for antiretroviral resistance analysis (1000 copies/ml). After extraction of HIV-1 RNA, a fragment of 1302 bp including the HIV-1 protease (PR) and the $5^{\prime}$-end of the reverse transcriptase (RT) open reading frames was amplified using the Viroseq HIV-1 Genotyping System (Abbott, Germany) following the manufacturer's instructions. The amplified fragments were directly sequenced with the ABI Prism 3100 automated sequencer (Applied Biosystems, Foster City, CA), data were analyzed with a dedicated software (Viroseq HIV-1 Genotyping System Software v2.5, Abbott, Germany) and manually edited where necessary.

\subsection{Phylogenetic analysis, subtyping and genetic distances}

Overall, all HIV-1 pol sequences obtained were firstly analysed using the NCBI Genotyping tool [http://www.ncbi.nlm.nih.gov/ projects/genotyping/formpage.cgi] in order to evaluate the similarity scores to specific HIV-1 subtypes. Then, nucleotide sequences were aligned using ClustalW software v2.0.9 (Larkin et al., 2007; Thompson et al., 1994) followed by minor manual adjustments using the BioEdit Software v7.0.9.0 (Hall, 1999). The final dataset included HIV-1 pol Sicilian sequences, as well as subtype-specific and CRF sequences downloaded from the HIV Los Alamos Database [http://www.hiv.lanl.gov/content/index].

HIV-1 subtype classification was performed by phylogenetic analysis. For this purpose, phylogenetic trees were built with the neighbor-joining (NJ) method implemented in the software MEGA v5.0.3 (Saitou and Nei, 1987; Tamura et al., 2011) according to the Tamura-Nei model of evolution and the $\gamma$ distribution of substitution rates among sites. The jModelTest 2 program (Darriba et al., 2012) was used to choose the appropriate nucleotide substitution for our dataset, the shape parameter $\alpha$ and the transition:transversion (T:t) ratio were calculated with Tree-Puzzle v5.2 (Schmidt et al., 2002), whereas the statistical robustness of the $\mathrm{NJ}$ trees and reliability of the branching orders was assessed with 1000 bootstrap resampling.
Phylogenetic network trees were also constructed with SplitsTree (Bryant and Moulton, 2004; Huson and Bryant, 2006) using the general time reversible nucleotide substitution model (GTR), with $\gamma$-distributed among-site rate heterogeneity.

All of the sequences were further investigated through both the web-based HIV-1 REGA Genotyping Tool v2.0 (de Oliveira et al., 2005) and the Simplot v3.5.1 software (Lole et al., 1999; Salminen et al., 1995) (sliding window: 200-nt, T:t ratio $=2.0$, model of evolution: Kimura two-parameter, bootstrap: 1000 replicates) to determine whether they were pure subtype or CRFs and to identify the recombination breakpoints. In this latter case, each "query" sequence was preliminarily compared with all major subtype/subsubtype "pure" reference sequences, and then run against only the strains involved in the recombination events to generate bootscan graphs. In any case, the fragments encompassed between breakpoints were confirmed through phylogenetic analyses, although almost all were too short to give a reliable phylogenetic signal. Alternative reference datasets were used in order to test the robustness of our findings (Table S1).

Finally, to identify the greater similarity of the study sequence to those stored in the international databases a BLAST search was conducted [http://blast.ncbi.nlm.nih.gov/Blast.cgi].

\subsection{Statistical analysis}

The clinical and epidemiological features of this group of HAART-naïve HIV-1 positive patients were compared to test the differences between subtype $B$ and non-B infected individuals by the $\chi^{2}$ test, Fisher exact test, or Wilcoxon test, as appropriate.

Cochran-Armitage test for trend was used to compare prevalences accross different sub-groups. Univariate and multivariate logistic regression analyses were performed including basic demographics (sex, age, ethnicity, and calendar years), immunological $\left(\mathrm{CD}_{4}^{+}\right.$and $\left.\mathrm{CD}_{8}^{+}\right)$, and virological (HIV viral load) parameters, as covariates.

Two sides $p$-values $<0.05$ were considered to be statistically significant. Data analyses were performed with STATA v12.1 MP for Macintosh (Apple) (StataCorp, 2011).

\subsection{Sequence data}

All 155 nucleotide sequences of HIV-1 pol gene reported in this study have been submitted to GenBank. HIV-1 sequences collected during the period 2004-2008 have been previously submitted under the following accession numbers: EF192302, EF192305, and GU969472-GU969580. The accession numbers HQ667668HQ667711 indicate new submissions and refer to nucleotide sequences obtained in 2009s.

\section{Results}

\subsection{Main characteristics of the study population}

Table 1 shows the epidemiological and clinical characteristics of the study population distributed in two different groups sustained by $B$ and non-B HIV-1 variant.

On a total of 155 HIV-1 HAART-naïve patients, 107 (69.0\%) were infected with B strains, whereas non-B subtypes were detected in 48 subjects (31.0\%).

During the study period, the increasing number of HIV-1 naïve patients per year, appeared consistent with a similar trend in the proportion of non-B variants (range: $7.8-40.9 \%, p=0.025$ ) and inversely correlated to the detection of B strains.

Male gender was prevalent in all groups and age-specific distribution (described in quartiles) showed a lower median age among 
Table 1

Epidemiological, clinical, and virological characteristics of the 155 HIV-1 HAART-naïve patients.

\begin{tabular}{|c|c|c|c|}
\hline \multirow[t]{2}{*}{ Characteristic } & \multirow[t]{2}{*}{ Total (\% by column) } & \multicolumn{2}{|c|}{ HIV-1 variants (\% by row) } \\
\hline & & B & Non-B \\
\hline \multicolumn{4}{|c|}{ Distribution by year of HIV diagnosis or entering the cohort $\left[n^{\circ}(\%)\right]$} \\
\hline Total & $155(100.0)$ & $107(69.0)$ & $48(31.0)$ \\
\hline 2004-2006 & $26(16.8)$ & $24(92.3)$ & $2(7.8)$ \\
\hline 2007 & $27(17.4)$ & $22(81.5)$ & $5(18.5)$ \\
\hline 2008 & $58(37.4)$ & $35(60.3)$ & $23(39.7)$ \\
\hline 2009 & $44(28.4)$ & $26(59.1)$ & $18(40.9)$ \\
\hline \multicolumn{4}{|l|}{ Gender $\left[n^{\circ}(\%)\right]$} \\
\hline Male & $112(72.3)$ & $86(76.8)$ & $26(23.2)$ \\
\hline Female & $43(27.7)$ & $21(48.8)$ & $22(51.1)$ \\
\hline \multicolumn{4}{|l|}{ Age group [years, $\left.n^{\circ}(\%)\right]$} \\
\hline$\leqslant 26$ & $40(25.8)$ & $22(55.0)$ & $18(45.0)$ \\
\hline $27-34$ & $38(24.5)$ & $27(71.1)$ & $11(28.9)$ \\
\hline $35-42$ & $41(26.5)$ & $28(68.3)$ & $13(31.7)$ \\
\hline$>42$ & $36(23.2)$ & $30(83.4)$ & $6(16.6)$ \\
\hline Age [years, median (IQR)] & $34.0(16.0)$ & $38.0(14.0)$ & $30.5(18.0)$ \\
\hline \multicolumn{4}{|l|}{ Route of infection $\left[n^{\circ}(\%)\right]$} \\
\hline Sexual & $149(96.1)$ & $104(69.8)$ & $45(30.2)$ \\
\hline Heterosexual & $92(59.4)$ & $51(55.4)$ & $41(44.6)$ \\
\hline Homo-bisexual & $57(36.8)$ & $53(93.0)$ & $4(7.0)$ \\
\hline Vertical (mother-to-child) & $4(2.6)$ & $1(25.0)$ & $3(75.0)$ \\
\hline Other/Unknown & $2(1.3)$ & $2(100.0)$ & $0(0)$ \\
\hline \multicolumn{4}{|l|}{ Geographic origin $\left[n^{\circ}(\%)\right]$} \\
\hline Italy & $113(72.9)$ & $102(90.3)$ & $11(9.7)$ \\
\hline Eastern Europe & $4(2.6)$ & $2(50.0)$ & $2(50.0)$ \\
\hline Africa & $38(24.5)$ & $3(7.9)$ & $35(92.1)$ \\
\hline Viral load $\left[\left(\log _{10}\right.\right.$ HIV-RNA copies $\left./ m L\right)$, median $\left.(I Q R)\right]$ & $5.0(1.3)$ & $4.9(1.3)$ & $5.3(1.2)$ \\
\hline $\mathrm{CD} 4^{+}$cell count $\left[\left(\right.\right.$cells $\left./ \mathrm{mm}^{3}\right)$, median $\left.(\mathrm{IQR})\right]$ & $281.5(379.0)$ & $280.0(413.0)$ & $283.0(244.0)$ \\
\hline$\leqslant 350$ cells $/ \mathrm{mm}^{3}\left[\mathrm{n}^{\circ}(\%)\right]$ & $94(60.7)$ & $62(66.0)$ & $32(34.0)$ \\
\hline$>350$ cells $/ \mathrm{mm}^{3}\left[\mathrm{n}^{\circ}(\%)\right]$ & $61(39.3)$ & $45(73.8)$ & $16(26.2)$ \\
\hline
\end{tabular}

IQR: interquartile range.

non-B infected patients (30.5 years), with a significant proportion of younger subjects ( $n=18 / 48 ; 37.5 \%)$, also confirmed by a logistic regression analysis $\left(\mathrm{OR}=4.1 ; \mathrm{Cl}_{95 \%}: 1.4-11.9\right)$.

In our study population, HIV-1 infection was mostly acquired through sexual intercourse (96.1\%), either heterosexual (59.4\%) or homo-bisexual (36.8\%). However, non-B subtype viruses were greatly represented among patients who acquired HIV-1 infection through heterosexual contacts $(85.4 \%, n=41 / 48)$.

Only $9.7 \%(n=11 / 113)$ of Italian-born subjects were infected with non-B HIV-1 variants. This latter group mainly consisted of male subjects (10 males and 1 female, respectively) with a median age of 36 years (IQR $=13$ years); $63.6 \%$ of them $(n=7 / 11)$ reported heterosexual promiscuity as risk factor, while $36.4 \%(n=4 / 11)$ were homosexual men. Moreover, all of these patients were included during the last 3-year period of the study, most of them (90.9\%, $n=10 / 11$ ) equally distributed in 2008s and 2009s.

Furthermore, $24.5 \%$ of study population ( $n=38 / 155)$ were African individuals, $92.1 \%$ of them $(n=35 / 38)$ harboured non-B viral strains. The strong association between the detection of non-B strains and the African origin of subjects was also supported by a logistic regression analysis, regardless of the HIV-1 transmission route $(\mathrm{OR}=11.7, p=0.035$ and $\mathrm{OR}=35.7, p=0.024$ for Africa and sub-Saharan Africa alone, respectively).

Both HIV-1 viral loads and $\mathrm{CD}_{4}^{+}$cell counts were quite similar in the two groups considered, although two-thirds of non-B patients had $\mathrm{CD}_{4}^{+}$cell counts lower than 350 cells $/ \mathrm{mm}^{3}$.

\subsection{Distribution of HIV-1 group $M$ variants in Sicily}

All of the 48 HIV-1 non-B sequences initially detected through the NCBI Genotyping tool were further analysed phylogenetically (Fig. 1). Overall, the neighbour-net tree generated with the
SplitsTree software assigned fourty-five sequences into four main clades (subtype C, F1, G, and CRF02_AG), while three single HIV-1 sequences clustered with CRF12_BF, CRF01_AE, and CRF09_cpx references, respectively.

Although the most part of pol sequences unambiguously felt within specific subtype radiations, the uncertain phylogenetic placement of some viral sequences suggested divergent evolutionary pathways in respect of their common ancestors (e.g., CV1188_79/08, CV1202_96/08, CV1361_143/09, and so on). Furthermore, each non-B nucleotide sequence was analysed using both REGA HIV-1 subtyping tool v2.0 and Simplot software (graphs available upon request) in order to validate the classification and to better explore distinct recombination breakpoints.

Bootscanning plots confirmed $66.7 \%(n=32 / 48)$ of these strains as non-B subtype or CRFs, while the remaining $(n=16 / 48)$ showed complex genetic patterns (Fig. 2).

CRF02_AG contributed in 28/48 strains (58.3\%), six of which included the CRF43_02G in their genetic organization (Fig. 2, Section 1).

Subtype F1 or F1-derived CRFs correlated to 4/48 (8.3\%) strains (Fig. 2, Section 2), while only two sequences were ascribed to CRF01_AE and CRF09_cpx, respectively (Fig. 2, Section 3).

Fourteen out of 48 (29.2\%) non-B infected patients were subtype C-related (Fig. 2, Section 4). In order to investigate the geographic origin of these strains, a maximum likelihood tree was generated from a dataset including both the $\mathrm{C}$-related pol sequences reported in this study and a group of reference sequences representative of the geographic areas endemic for subtype $C$ infections such as South Africa, Ethiopia, Djbouti, and Brazil (Fig. 3).

Nine out of 14 subtype C-related strains here reported clustered with HIV-1 reference sequences from the "Horn of Africa", in accordance to the geographic origin of the infected patients, while 


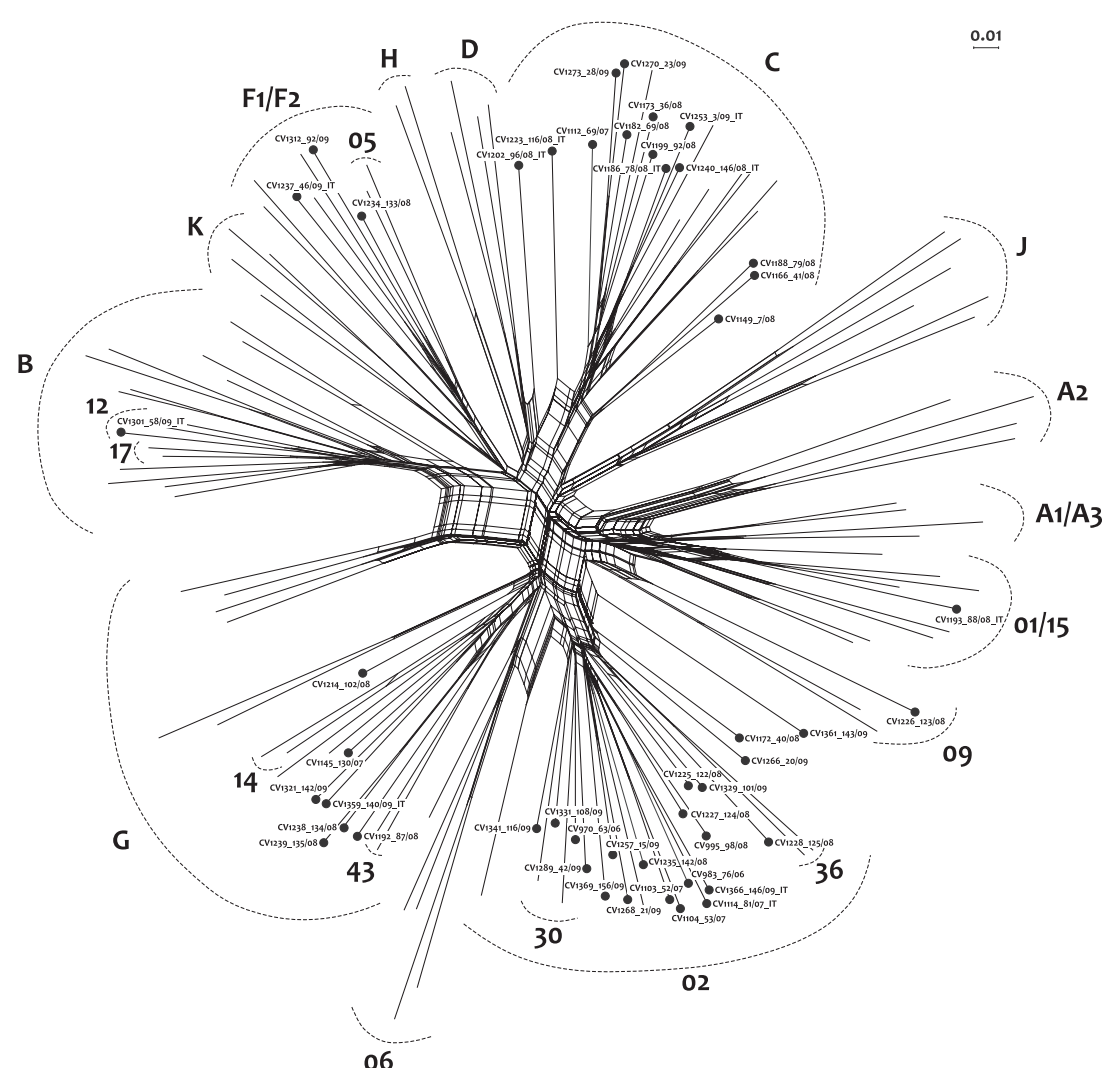

$A_{1} / A_{3}$

Fig. 1. SplitTree analysis of the 48 HIV-1 Sicilian non-B sequences. Neighbor-net tree describing the phylogenetic relationships of non-B aligned nucleotide sequences representing the protease and reverse transcriptase in the pol gene. The splits graph was constructed using NeighborNet methodology with pairwise distance input, which was estimated by GTR distance and g-distributed among-site heterogeneity. All samples characterized in this study are labeled on the corresponding branch. The "IT" suffix is used to indicate sequences from Italian subjects.

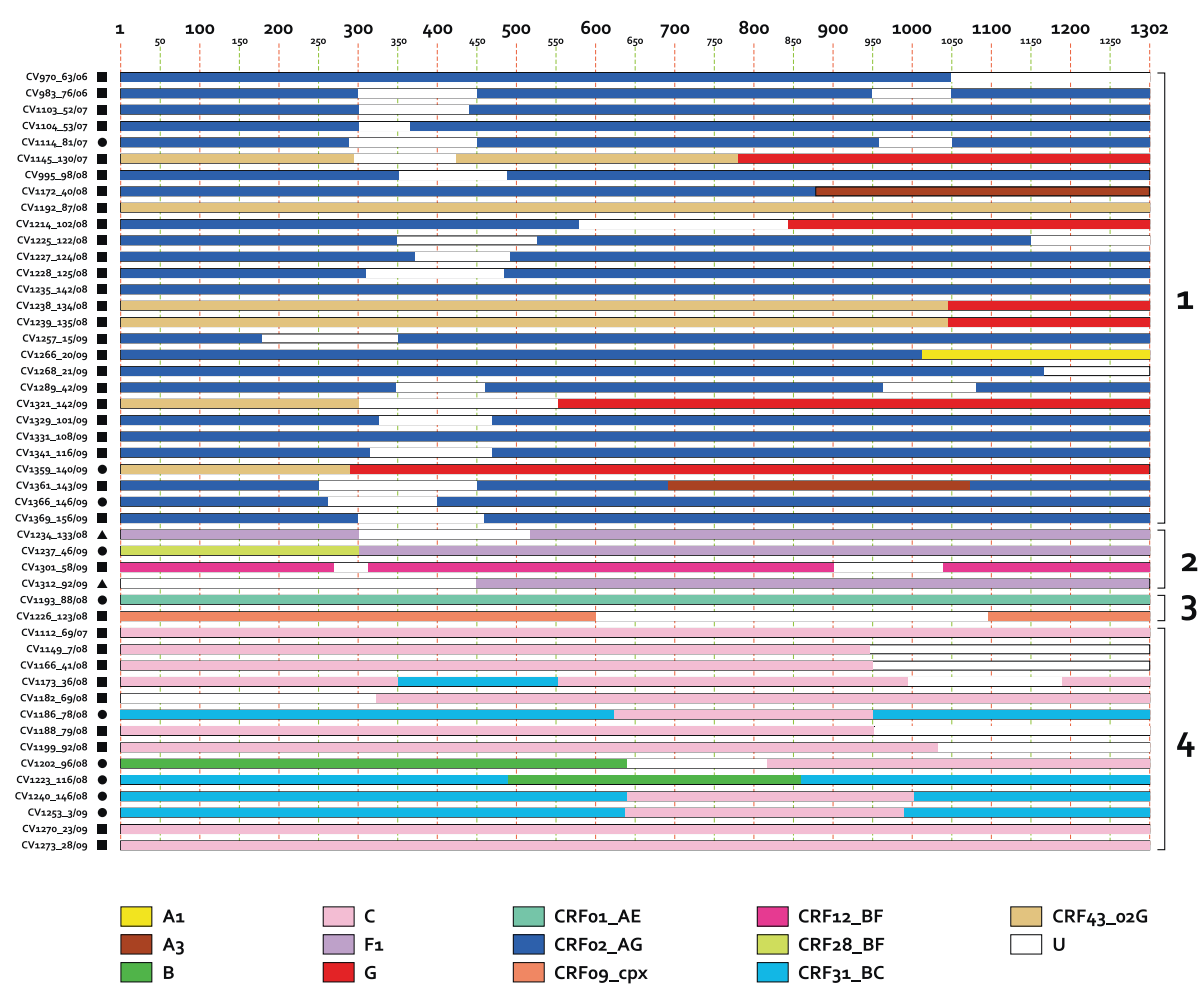

Fig. 2. Schematic representation of the genetic structure of HIV-1 non-B pol sequences. Only fragments with bootstrap values $\geqslant 70 \%$ are represented as known subtypes or CRFs. Geographic origin: Italy, Africa, $\triangle$ Eastern Europe. Section 1 includes CRF02_AG-related strains. Section 2 includes subtype F1-related strains. Section 3 includes one CRF01_AE sequence and one CRF09_Cpx sequence, respectively. Section 4 includes subtype C-related strains. 


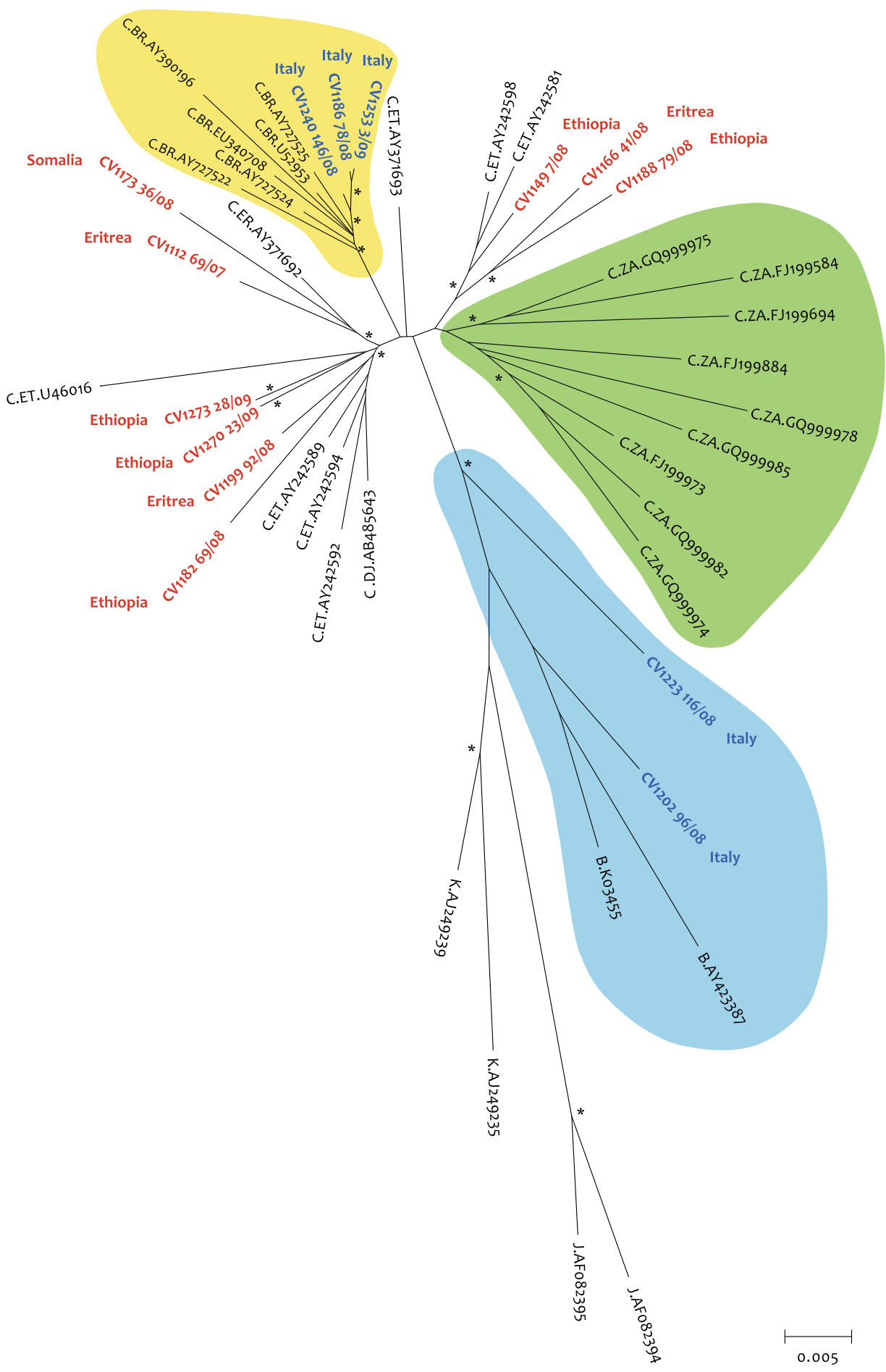

Fig. 3. Maximum likelihood phylogenetic relationships of HIV-1 subtype C-related pol sequences of HIV-1 infections in Sicily. Maximum likelihood phylogenetic tree constructed using 1,302 nucleotide sites (HXB2 coordinates: nt 2253-3554) of HIV-1 subtype C-related pol sequences from Sicily, Brazil, Horn of Africa, and South Africa. Green area clusters South African reference strains, blue area includes strains from Italian-born individuals, and yellow area includes strains from Italian-born individuals with similarity to Brazilian C-reference strains. Bootstrap values greater than $75 \%$ are indicated with $*$ on the branch leading to the reference strains. Clinical isolates are denoted with "CV" prefix and are indicated in bold. Each subtype C reference strain's label includes Subtype Country Genbank Accession number. Schematic representation of the mosaic pol fragments derived from bootscanning plots is shown in Fig. 2. BR: Brazil; DJ: Djbouti; ER: Eritrea; ET: Ethiopia; ZA: South Africa.

three sequences from Italian-born individuals grouped differently with a set of Brazilian strains. Finally, the remaining two sequences did not clearly fall into subtype $C$ radiation, one of which showed a divergent evolutionary pathway with the strongest similarity to both a single sequence described in France (Frange et al., 2008) and a group of viral sequences recently found in Southern Italy (Monno et al., 2012), and classified as BC recombinants.

Fig. 4 depicts the maximum likelihood tree describing the phylogenetic relationships between our BC recombinant sequence with those from Southern Italy and France, together with a set of 


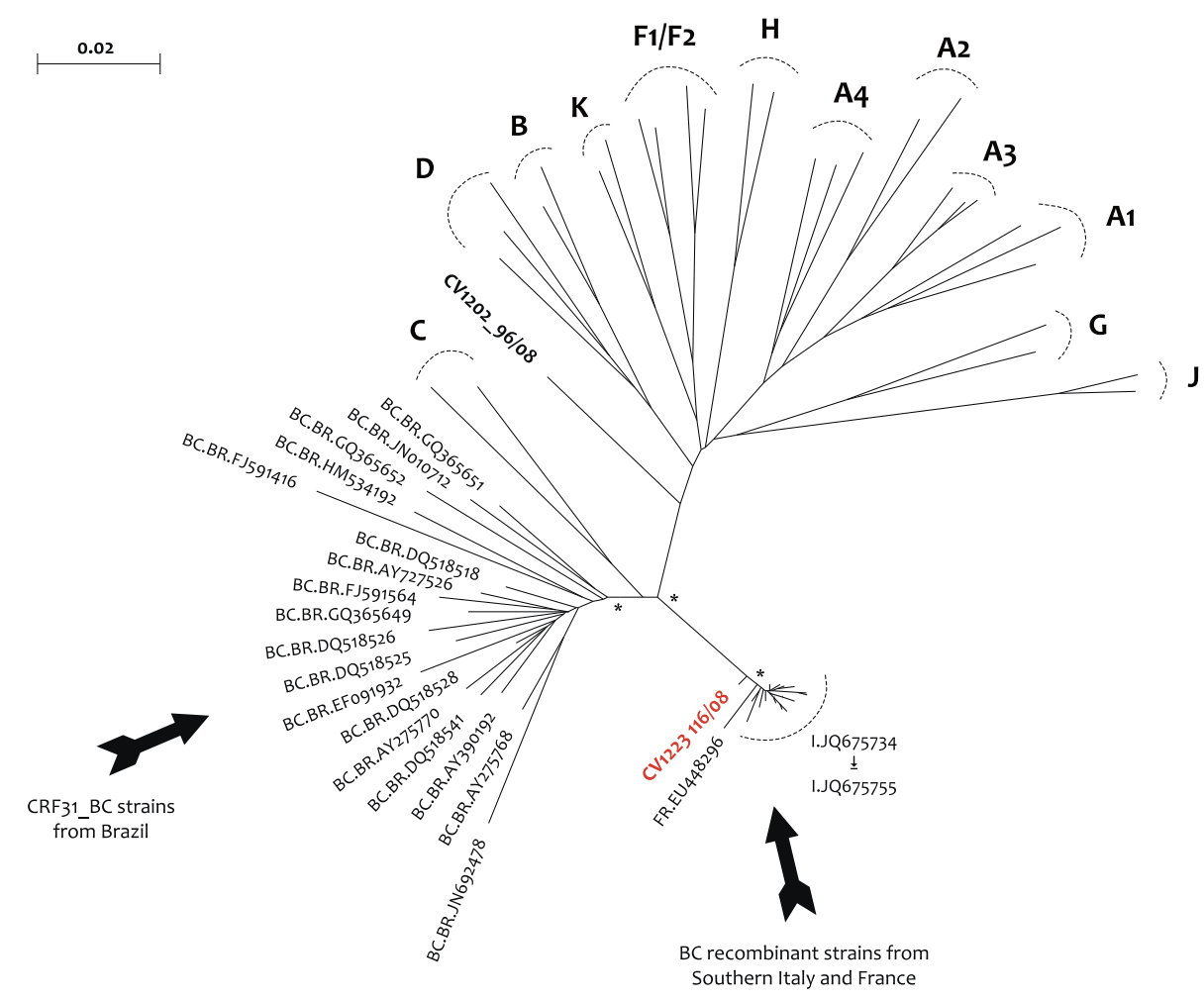

Fig. 4. Maximum likelihood tree constructed from HIV-1 BC recombinant pol sequences from the Mediterranean area and Brazilian relatives. Maximum likelihood phylogenetic tree constructed using 1302 nucleotide sites of pol sequences (nucleotide 2253-3554 of HXB2 coordinates) from 24 HIV-1 BC recombinants from the Mediterranean area. (Sicily, $n=1$; Apulia, $n=22$; France, $n=1$ ) and close relatives from Brazil. The Sicilian BC recombinant strain is denoted with "CV" prefix and coloured in red. Each CRF31_BC strain's label includes Subtype Country Genbank Accession number. Bootstrap values greater than $75 \%$ are indicated with * on the corresponding branch. BR: Brazil; I: Italy; F: France.

CRF31_BC strains from Brazil. Interestingly, all of these BC recombinants felt into an isolated monophyletic group sharing a common ancestor with the cluster of CRF31_BC Brazilian sequences.

\section{Discussion}

In the present work, 48/155 (31.0\%) HIV-1 viral sequences from HAART-naïve patients were phylogenetically analysed and classified as non-B strains. Although this result resemble those reported in other studies (Hemelaar et al., 2006), it demonstrates an increasing trend by year, and ultimately confirms the observations from other European countries with much tighter historical relationships with countries endemic for HIV-1 non-B infections (Holguín et al., 2008a,b; Semaille et al., 2007; Tatt et al., 2004; Vidal et al., 2008).

Different circumstances can offer a valid explanation for the introduction and spread of some specific non-B subtypes in the native population. Firstly, migration flow from countries with high prevalence of HIV to European geographic areas is dramatically increasing (Del Amo et al., 2011), with an annual increment of about $15 \%$ in Sicily, where more than $35.0 \%$ of immigrants originates from African countries (Caritas/Migrantes, 2009).

Secondly, in our country, these immigrants often live under conditions of poverty and deprivation. Consequently, prostitution and occasional sexual intercourses between local subjects and immigrant partners contribute to the spread of non-B subtypes in the native population (Holguín et al., 2008a), further charged by the low usage of male condom due in part to a lack of, or poor, knowledge of prevention practices and also to the perceived "low risk environment" of HIV transmission in European countries (Barrett and Mulugeta, 2010).
Thirdly, travelling to countries with easier opportunities for sexual promiscuity (i.e. Brazil, the Caribbean, and Thailand) could likely be responsible for the entry of an array of group M subtypes into formerly subtype B-restricted geographic areas and, consequently, contributing for the changing profile of the local HIV epidemiology in host countries (Rice et al., 2012). As similarly reported in other studies in Italy (Lai et al., 2010) and elsewhere (Hawke et al., 2012; von Wyl et al., 2011), in the present paper, the totality of non-B infections among immigrant subjects correlate to heterosexual transmission, while non-B infections in homosexual individuals were exclusively found in Italian-born patients. Of note, native Italians who were infected with HIV-1 non-B strains mainly harboured URFs while known CRFs/subtypes were found only in a minority group of subjects.

The present work confirms CRF02_AG as the prevalent circulating recombinant form in our region, as well as in several European countries (González-Alba et al., 2011; von Wyl et al., 2011; Yebra et al., 2012), in contrast to that observed in the northern part of Italy where subtype $\mathrm{F} 1$ is the most prevalent non-B clade (Lai et al., 2010), mirroring the extensive influx of immigrants from Romania, an European country with a massive prevalence of subtype F1 HIV-1 infections (Lai et al., 2012).

Although Romanians account for the main foreign community also in Sicily (Caritas/Migrantes, 2009), the low prevalence of subtype F1 strains in our series may has been limited by the restricted number of Eastern European immigrant subjects included in this study.

A second main group of non-B HAART-naïve patients was infected with subtype C-related HIV-1 strains. The phylogenetic evolutionary analysis evidenced how subtype $C$ infections in Sicily are mostly derived from travelling abroad or driven by immigrants from endemic areas of the African continent, also confirming the 
introduction of viral variants uncommon in our region as well as in Europe.

Intriguingly, a single C-related sequence with unresolved classification and belonging to a native Italian homosexual man, strongly correlated to a group of sequences described in Apulia (Monno et al., 2012) and in France (Frange et al., 2008), and closely related to those described by other authors in Brazil (Brígido et al., 2007; Gräf and Pinto, 2012; Passaes et al., 2009a,b; Santos et al., 2006).

Furthermore, the detection of such URFs-BC in three different geographic areas with no evidence of epidemiological link together with a local divergent evolutionary pathway, could represent a potential candidate for a novel CRF_BC profile, although a whole genome sequencing is mandatory to definitely confirm this evidence.

In summary, our findings suggest that the dynamics of HIV epidemic in Sicily seems to be driven by introducing more and more different non-B strains, some of which rare in our geographic area. Migration of several HIV-1 infected ethnic groups, mainly from Africa and Eastern Europe, mirrors in Sicily the epidemiology of HIV epidemics from their respective countries of origin. In addition, the detection of non-B subtypes in Italian-born subjects is compatible with sexual partners in/from countries with high endemicity and diversity for HIV.

The continuing increase of complexity and genetic diversity of the global HIV-1 pandemic represent a great challenge for the monitoring of the infection, either locally or globally. It could assume, in the future, important implications also in terms of diagnostic accuracy and sensitivity, efficacy of antiretroviral drugs, and may have serious consequences on efforts to control the AIDS pandemic with future vaccination trials (Holguín et al., 2006; Rouet et al., 2007; Wainberg, 2004).

For these reasons, continuous surveillance of HIV variants and evolutionary research studies are of paramount importance to a better implementation of tailored public health policies.

\section{Conflict of Interest}

The authors declare that they have no conflict of interest.

\section{Author contributions}

This project was developed by F.T. and F.V. Laboratory analyses were performed by F.T., CM.M., F.B., AM.P., and F.T. supervised the process. Finally, F.T. and F.V. analysed the data and wrote the manuscript. F.T. takes primary responsibility for this work, together with F.V. All authors read and approved the final manuscript.

\section{Funding}

This research received no specific grant from any funding agency in the public, commercial or not-for-profit sectors.

\section{Acknowledgment}

We thank James J. Goedert for his valuable comments and for the helpful review of this paper.

\section{Appendix A. Supplementary data}

Supplementary data associated with this article can be found, in the online version, at http://dx.doi.org/10.1016/j.meegid.2013. 02.012 .

\section{References}

Barrett, H.R., Mulugeta, B., 2010. Human Immunodeficiency Virus (HIV) and migran "risk environments": the case of the Ethiopian and Eritrean immigrant community in the West Midlands of the UK. Psychol. Health Med. 15, 357-369.

Brígido, L.F., Nunes, C.C., Oliveira, C.M., Knoll, R.K., Ferreira, J.L., Freitas, C.A., Alves, M.A., Dias, C., Rodrigues, R., Program, Research.Capacity., 2007. HIV type 1 subtype $C$ and $C B$ Pol recombinants prevail at the cities with the highest AIDS prevalence rate in Brazil. AIDS Res. Hum. Retroviruses 23, 1579-1586.

Bryant, D., Moulton, V., 2004. Neighbor-net: an agglomerative method for the construction of phylogenetic networks. Mol. Biol. Evol. 21, 255-265.

Buonaguro, L., Tagliamonte, M., Tornesello, M.L., Ciuffreda, D., Capiluppi, B., Lopalco L., Lazzarin, A., Tambussi, G., Buonaguro, F.M., 2002. Molecular and phylogenetic characterization of HIV variants in Italian primary HIV infections (PHI): identification of non-B subtype variants. J. Biol. Regul. Homeost. Agents 16, 44-48.

Caritas/Migrantes, 2009. Dossier Statistico - XIX Rapporto sull'immigrazione. IDOS, Rome.

Darriba, D., Taboada, G.L., Doallo, R., Posada, D., 2012. JModelTest 2: more models new heuristics and parallel computing. Nat. Meth. 9, 772. http://dx.doi.org/ 10.1038/nmeth.2109.

de Oliveira, T., Deforche, K., Cassol, S., Salminen, M., Paraskevis, D., Seebregts, C., Snoeck, J., van Rensburg, E.J., Wensing, A.M., van de Vijver, D.A., Boucher, C.A. Camacho, R., Vandamme, A.M., 2005. An automated genotyping system for analysis of HIV-1 and other microbial sequences. Bioinformatics 21, 3797-3800.

Del Amo, J., Likatavičius, G., Pérez-Cachafeiro, S., Hernando, V., González, C., Jarrín, I. Noori, T., Hamers, F.F., Bolúmar, F., 2011. The epidemiology of HIV and AIDS reports in migrants in the 27 European Union countries, Norway and Iceland: 1999-2006. Eur. J. Public Health 21, 620-626.

Frange, P., Galimand, J., Vidal, N., Goujard, C., Deveau, C., Souala, F., Peeters, M. Meyer, L., Rouzioux, C., Chaix, M.L., 2008. New and old complex recombinant HIV-1 strains among patients with primary infection in 1996-2006 in France. The French ANRS CO06 primo cohort study. Retrovirology 5, 69.

Geretti, A.M., 2006. HIV-1 subtypes: epidemiology and significance for HIV management. Curr. Opin. Infect. Dis. 19, 1-7.

González-Alba, J.M., Holguín, A., Garcia, R., García-Bujalance, S., Alonso, R., Suárez, A., Delgado, R., Cardeñoso, L., González, R., García-Bermejo, I., Portero, F., de Mendoza, C., González-Candelas, F., Galán, J.C., 2011. Molecular surveillance of HIV-1 in Madrid, Spain: a phylogeographic analysis. J. Virol. 85, 10755-10763.

Gräf, T., Pinto, A.R., 2012. The increasing prevalence of HIV-1 subtype C in Southern Brazil and its dispersion through the continent. Virology S0042-S6822 (12) 00441-00442. http://dx.doi.org/10.1016/j.virol.2012.08.048, pii: S00426822(12)00441-2.

Hall, T.A., 1999. BioEdit: a user-friendly biological sequence alignment editor and analysis program for Windows 95/98/NT. Nucl. Acids Symp. Ser. 41, 95-98.

Hawke, K.G., Waddell, R.G., Gordon, D.L., Ratcliff, R.M., Ward, P.R., Kaldor, J.M., 2012 HIV non-B subtype distribution: emerging trends and risk factors for imported and local infections newly diagnosed in South Australia. AIDS Res. Hum. Retroviruses, 25.

Hemelaar, J., Gouws, E., Ghys, P.D., Osmanov, S., 2006. Global and regional distribution of HIV-1 genetic subtypes and recombinants in 2004. AIDS 20, W13-W23.

Holguín, A., Ramirez de Arellano, E., Rivas, P., Soriano, V., 2006. Efficacy of antiretroviral therapy in individuals infected with HIV-1 non-B subtypes. AIDS Rev. 8, 98-107.

Holguín, A., de Mulder, M., Yebra, G., López, M., Soriano, V., 2008a. Increase of non-B subtypes and recombinants among newly diagnosed HIV-1 native Spaniards and immigrants in Spain. Curr. HIV Res. 6, 327-334.

Holguín, A., Lospitao, E., López, M., de Arellano, E.R., Pena, M.J., del Romero, J. Martín, C., Soriano, V., 2008b. Genetic characterization of complex interrecombinant HIV-1 strains circulating in Spain and reliability of distinct rapid subtyping tools. J. Med. Virol. 80, 383-391.

Huson, D.H., Bryant, D., 2006. Application of phylogenetic networks in evolutionary studies. Mol. Biol. Evol. 23, 254-267.

Lai, A., Riva, C., Marconi, A., Balestrieri, M., Razzolini, F., Meini, G., Vicenti, I., Rosi, A Saladini, F., Caramma, I., Franzetti, M., Rossini, V., Galli, A., Galli, M., Violin, M. Zazzi, M., Balotta, C., 2010. Changing patterns in HIV-1 non-B clade prevalence and diversity in Italy over three decades. HIV Med. 11, 593-602.

Lai, A., Simonetti, F.R., Zehender, G., De Luca, A., Micheli, V., Meraviglia, P., Corsi, P., Bagnarelli, P., Almi, P., Zoncada, A., Paolucci, S., Gonnelli, A., Colao, G., Tacconi, D., Franzetti, M., Ciccozzi, M., Zazzi, M., Balotta, C., 2012. HIV-1 subtype F1 epidemiological networks among Italian heterosexual males are associated with introduction events from South America. PLoS One 7, e42223.

Larkin, M.A., Blackshields, G., Brown, N.P., Chenna, R., McGettigan, P.A., McWilliam, H., Valentin, F., Wallace, I.M., Wilm, A., Lopez, R., Thompson, J.D., Gibson, T.J., Higgins, D.G., 2007. Clustal $W$ and Clustal X version 2.0. Bioinformatics 23 , 2947-2948

Liu, Y., Li, L., Bao, Z., Li, H., Zhuang, D., Liu, S., Wang, X., Li, T., Jia, L., Yang, S., Li, J., 2012. Identification of a novel HIV type 1 circulating recombinant form (CRF52 01B) in Southeast Asia. AIDS Res. Hum. Retroviruses 28, 1357-1361.

Lole, K.S., Bollinger, R.C., Paranjape, R.S., Gadkari, D., Kulkarni, S.S., Novak, N.G., Ingersoll, R., Sheppard, H.W., Ray, S.C., 1999. Full-length human immunodeficiency virus type 1 genomes from subtype C-infected 
seroconverters in India, with evidence of intersubtype recombination. J. Virol. $73,152-160$

Monno, L., Brindicci, G., Lo Caputo, S., Punzi, G., Scarabaggio, T., Riva, C., Di Bari, C., Pierotti, P., Saracino, A., Lagioia, A., Mazzotta, F., Balotta, C., Angarano, G., 2005. HIV-1 subtypes and circulating recombinant forms (CRFs) from HIV-infected patients residing in two regions of central and southern Italy. J. Med. Virol. 75, 483-490.

Monno, L., Brindicci, G., Lai, A., Punzi, G., Altamura, M., Simonetti, F.R., Ladisa, N., Saracino, A., Balotta, C., Angarano, G., 2012. An outbreak of HIV-1 BC recombinants in Southern Italy. J. Clin. Virol. 55, 370-373.

Ng, O.T., Eyzaguirre, L.M., Carr, J.K., Chew, K.K., Lin, L., Chua, A., Leo, Y.S., Redd, A.D., Quinn, T.C., Laeyendecker, O., 2012. Identification of new CRF51_01B in Singapore using full genome analysis of three HIV type 1 isolates. AIDS Res. Hum. Retroviruses 28, 527-530.

Passaes, C.P., Bello, G., Lorete, R.S., Matos Almeida, S.E., Junqueira, D.M., Veloso, V.G., Morgado, M.G., Guimarães, M.L., 2009a. Genetic characterization of HIV-1 BC recombinants and evolutionary history of the CRF31_BC in Southern Brazil. Infect. Genet. Evol. 9, 474-482.

Passaes, C.P., Guimarães, M.L., Bello, G., Morgado, M.G., 2009b. Near full-length genome characterization of HIV type 1 unique $\mathrm{BC}$ recombinant forms from Southern Brazil. AIDS Res. Hum. Retroviruses 25, 1339-1344.

Peeters, M., Toure-Kane, C., Nkengasong, J.N., 2003. Genetic diversity of HIV in Africa: impact on diagnosis, treatment, vaccine development and trials. AIDS 17, 2547-2560.

Rice, B., Gilbart, V.L., Lawrence, J., Smith, R., Kall, M., Delpech, V., 2012. Safe travels? HIV transmission among Britons travelling abroad. HIV Med. 13, 315317.

Rouet, F., Chaix, M.L., Nerrienet, E., Ngo-Giang-Huong, N., Plantier, J.C., Burgard, M., Peeters, M., Damond, F., Ekouevi, D.K., Msellati, P., Ferradini, L., Rukobo, S., Maréchal, V., Schvachsa, N., Wakrim, L., Rafalimanana, C., Rakotoambinina, B., Viard, J.P., Seigneurin, J.M., Rouzioux, C., 2007. Impact of HIV-1 genetic diversity on plasma HIV-1 RNA Quantification: usefulness of the Agence Nationale de Recherches sur le SIDA second-generation long terminal repeat-based real-time reverse transcriptase polymerase chain reaction test. J. Acquir. Immune Defic. Syndr. 45, 380-388.

Ruchansky, D., Casado, C., Russi, J.C., Arbiza, J.R., Lopez-Galindez, C., 2009. Identification of a new HIV Type 1 circulating recombinant form (CRF38_BF1) in Uruguay. AIDS Res. Hum. Retroviruses 25, 351-356.

Saitou, N., Nei, M., 1987. The neighbor-joining method: a new method for reconstructing phylogenetic trees. Mol. Biol. Evol. 4, 406-425.

Salminen, M.O., Carr, J.K., Burke, D.S., McCutchan, F.E., 1995. Identification of breakpoints in intergenotypic recombinants of HIV type 1 by bootscanning. AIDS Res. Hum. Retroviruses 11, 1423-1425.

Santos, A.F., Sousa, T.M., Soares, E.A., Sanabani, S., Martinez, A.M., Sprinz, E., Silveira, J., Sabino, E.C., Tanuri, A., Soares, M.A., 2006. Characterization of a new circulating recombinant form comprising HIV-1 subtypes C and B in southern Brazil. AIDS 20, 2011-2019.
Schmidt, H.A., Strimmer, K., Vingron, M., von Haeseler, A., 2002. TREE-PUZZLE: maximum likelihood phylogenetic analysis using quar- tets and parallel computing. Bioinformatics $18,502-504$.

Semaille, C., Barin, F., Cazein, F., Pillonel, J., Lot, F., Brand, D., Plantier, J.C., Bernillon, P., Le Vu, S., Pinget, R., Desenclos, J.C., 2007. Monitoring the dynamics of the HIV epidemic using assays for recent infection and serotyping among new HIV diagnoses: experience after 2 years in France. J. Infect. Dis. 196, 377-383.

StataCorp, 2011. Stata Statistical Software: Release 12. StataCorp LP, College Station (TX).

Tamura, K., Peterson, D., Peterson, N., Stecher, G., Nei, M., Kumar, S., 2011. MEGA5: molecular evolutionary genetics analysis using maximum likelihood, evolutionary distance, and maximum parsimony methods. Mol. Biol. Evol. 28, 2731-2739.

Tatt, I.D., Barlow, K.L., Clewley, J.P., Gill, O.N., Parry, J.V., 2004. Surveillance of HIV-1 subtypes among heterosexuals in England and Wales, 1997-2000. J. Acquir. Immune Defic. Syndr. 36, 1092-1099.

Thompson, J.D., Higgins, D.G., Gibson, T.J., 1994. CLUSTAL W: improving the sensitivity of progressive multiple sequence alignment through sequence weighting, positions-specific gap penalties and weight matrix choice. Nucleic Acids Res. 22, 4673-4680.

Thomson, M.M., Nájera, R., 2005. Molecular epidemiology of HIV-1 variants in the global AIDS pandemic: an update. AIDS Rev. 7, 210-224.

Tramuto, F., Vitale, F., Bonura, F., Romano, N., 2004. For the Group for HIV-1 Antiretroviral Studies in Sicily Detection of HIV type 1 non-B subtypes in Sicily, Italy. AIDS Res. Hum. Retroviruses 20, 251-254.

Tramuto, F., Bonura, F., Perna, A.M., Mancuso, S., Firenze, A., Romano, N., Vitale, F., 2007. For the Group for HIV-1 Antiretroviral Studies in Sicily Genetic diversity of HIV-1 non-B strains in Sicily evidence of intersubtype recombinants by sequence analysis of gag, pol, and env genes. AIDS Res. Hum. Retroviruses 23, 1131-1138.

Vidal, N., Frange, P., Chaix, M.L., Mulanga, C., Lepira, F., Bazepeo, S.E., Goujardm, C., Meyer, L., Rouzioux, C., Delaporte, E., Peeters, M., 2008. Characterization of an old complex circulating recombinant form, CRF27_cpx, originating from the Democratic Republic of Congo (DRC) and circulating in France. AIDS Res. Hum. Retroviruses 24, 315-321.

von Wyl, V., Kouyos, R.D., Yerly, S., Böni, J., Shah, C., Bürgisser, P., Klimkait, T., Weber, R., Hirschel, B., Cavassini, M., Staehelin, C., Battegay, M., Vernazza, P.L., Bernasconi, E., Ledergerber, B., Bonhoeffer, S., Günthard, H.F., 2011. Swiss HIV Cohort Study The role of migration and domestic transmission in the spread of HIV-1 non-B subtypes in Switzerland. J. Infect. Dis. 204, 1095-1103.

Wainberg, M.A., 2004. HIV-1 subtype distribution and the problem of drug resistance. AIDS Suppl. 3, S63-S68.

Yebra, G., de Mulder, M., Martín, L., Rodríguez, C., Labarga, P., Viciana, I., Berenguer, J., Alemán, M.R., Pineda, J.A., García, F., Holguín, A., 2012. Cohort of the Spanish AIDS Research Network (CoRIS) Most HIV type 1 non-B infections in the Spanish cohort of antiretroviral treatment-naïve HIV-infected patients (CoRIS) are due to recombinant viruses. J. Clin. Microbiol. 50, 407-413. 